\title{
Hardware implementation of SPWM technique for an Inverter
}

\author{
Preethi Balaji ${ }^{1}$, Madhuri ${ }^{2}$, Payal Patro ${ }^{3}$, Jaybhushan. ${ }^{4}$, Supriya $\mathrm{P}^{5}$ \\ 1, 2, 3, 4, 5 (Electrical Engineering, BNMIT Bangalore, India)
}

\begin{abstract}
Due to large application of induction motor in industries it has become prudent to work on its efficiency. As the inverter output provides variable voltage magnitude and frequency, with lesser harmonics the efficiency of induction motor is improved. It is possible only with a better gate pulses. With recent development in power electronic devices, various PWM techniques have been developed. The most widely used PWM technique for a 3 phase VSI is SPWM. This paper focuses on step by step development, simulation using MATLAB/SIMULINK and also hardware implementation of SPWM pulses.
\end{abstract}

Keywords: Matlab/Simulink

\section{Introduction}

Alternating current (AC) power can be achieved from direct current (DC) power at desired output current or voltage and frequency through inversion. Voltage-fed and current-fed are commonly used terms in inverter circuits. In voltage source inverter (VSI) DC input voltage is normally constant and it is also not dependent on the load current which is drawn from it. The load voltage is decided by inverter and waveform of current is determined by the load. Normally machines do not perform smoothly due to large amount of \%THD, which causes noise, vibration and heating in machines. Enough amount of research has been done already in this area suggesting different techniques for reducing the harmonics in a machine. The purpose of this paper is to give a detailed design of SPWM techniques for control of inverter gate pulse.

\subsection{Modulation Technique:}

The fundamental idea of the PWM technique is to compare a high frequency wave or signal known as the carrier signal (a triangular signal with frequency $f_{s}$ ) to a signal of low frequency known as the reference modulating signal ( with frequency $\mathrm{f}_{\mathrm{m}}$ ). The frequency of the reference-modulating signal $\mathrm{f}_{\mathrm{m}}$ is set the desired output frequency. In Sinusoidal Pulse width modulation technique for getting the pulses, it is required to compare sine wave with triangular wave. The carrier - based PWM technique fulfils such a requirement as it defines the on and off states of the switches of one leg of a VSI by comparing a modulating signal $v \boldsymbol{c}$ (desired ac output voltage) and a triangular waveform $\boldsymbol{v} \Delta$ (carrier signal). In practice, when $\boldsymbol{v} \boldsymbol{c}>\boldsymbol{v} \Delta \boldsymbol{t h e}$ switch $\mathrm{S}+$ is on and the switch S- is off; similarly, when $v \boldsymbol{c}<\boldsymbol{v} \Delta$ the switch S+ is off and the switch S- is on. A special case is when the modulating signal $v \boldsymbol{c}$ is a sinusoidal at frequency $f \boldsymbol{c}$ and amplitude $v \boldsymbol{c}$, and the triangular signal $\boldsymbol{v} \Delta$ is at frequency $\boldsymbol{f} \Delta$ and amplitude $\boldsymbol{v} \Delta$. This is the sinusoidal PWM (SPWM) scheme. In this case, the modulation index $\boldsymbol{m a}$ (also known as the amplitude - modulation ratio).

$$
\begin{aligned}
& m_{a}=\frac{\hat{v}_{c}}{\hat{v}_{\Delta}} \\
& m_{f}=\frac{f_{\Delta}}{f_{c}}
\end{aligned}
$$

Additional important issues are:

a) For small values of $\boldsymbol{m} \boldsymbol{f}(\boldsymbol{m} \boldsymbol{f}<21)$, the carrier signal $\boldsymbol{v}_{\Delta}$ and the modulating signal $\boldsymbol{v} \boldsymbol{c}$ should be synchronized to each other ( $\boldsymbol{m} \boldsymbol{f}$ integer), which is required to hold the previous features; if this is not the case,

sub harmonics will be present in the ac output voltage.

b) For large values of $\boldsymbol{m} \boldsymbol{f}(\boldsymbol{m} \boldsymbol{f}>21)$, the sub harmonics are negligible if an asynchronous PWM technique is used, however, due to potential very low-order sub harmonics, its use should be avoided.

c) In the over modulation region $(\boldsymbol{m a}>1)$ some intersections between the carrier and the modulating signal are missed, which leads to the generation of low-order harmonics but a higher fundamental ac output voltage is obtained; unfortunately, the linearity between $\mathbf{m a} \mathbf{a n d} \boldsymbol{v}^{\wedge} \mathbf{0 1}$ achieved in the linear region. 


\subsection{SPWM Technique for three phase VSI}

This is an extension of the one introduced for single-phase VSIs. In this case and in order to produce $120^{\circ}$ out-of-phase load voltages, three modulating signals that are $120^{\circ}$ out of phase are used. Carrier signal and preserve the features of the PWM technique, the normalized carrier frequency mf should be an odd multiple of 3. Thus, all phase voltages ( $\mathrm{vaN}, \mathrm{vbN}$, and $\mathrm{vcN}$ ) are identical but $1200^{\circ}$ out of phase without even harmonics; moreover, harmonics at frequencies a multiple of 3 are identical in amplitude and phase in all phases.

\section{Software implementation:}

\subsection{Simulink Model of SPWM technique}

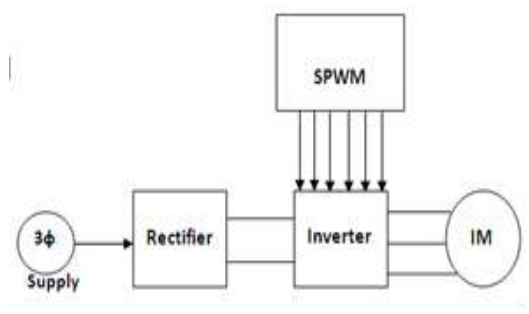

Fig1. Overall block diagram.

The Simulink results are shown at Switching frequency $\left(\mathrm{f}_{\mathrm{s}}\right)=3 \mathrm{kHz}$, DC voltage $\left(\mathrm{V}_{\mathrm{dc}}\right)=352 \mathrm{~V}$, Frequency of reference wave $(\mathrm{f})=50 \mathrm{~Hz}$, Modulation index $(\mathrm{M})=1$

Quality means what \% of harmonic of its fundamental is present in line voltage or current which is provided to the stator to the motor. Hence \% THD (total harmonics distortion) determines which output voltage is more close to sine wave.

\subsection{Simulink Results}
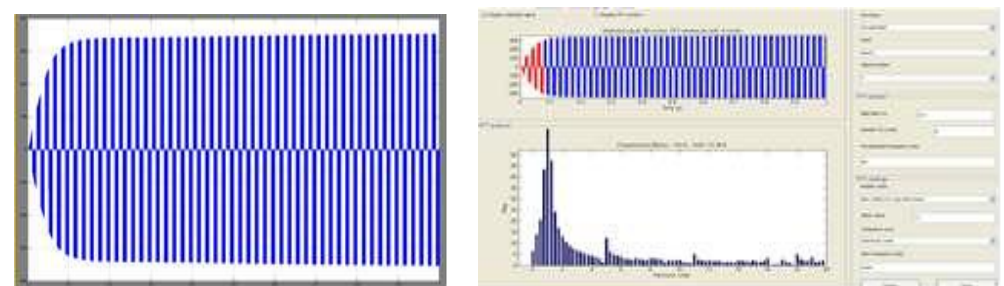

Fig2. Output of line voltage of SPWM technique

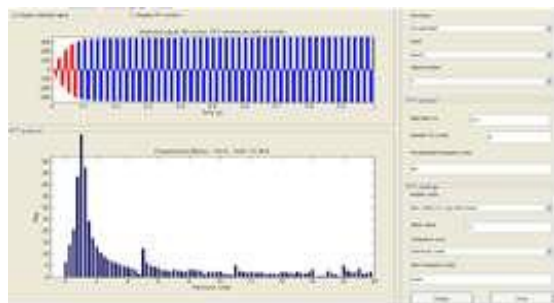

Fig3. FFT analysis with \% THD Output of line Voltage of SPWM technique
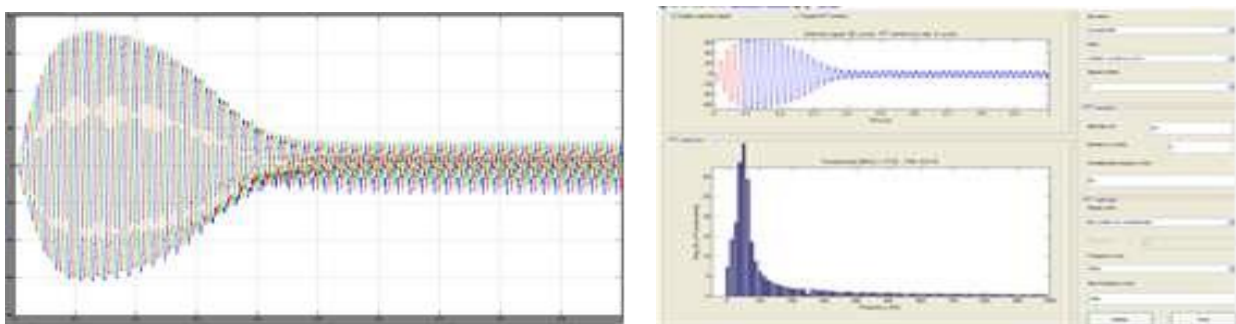

Fig4. 3-phase stator current of SPWM Fig5. FFT analysis with \%THD in stator current of SPWM technique. 


\section{Hardware implementation:}

The hardware implementation for the following circuits below is discussed in detail.

- $\quad$ Power supply circuit

- Control circuit

- Isolation circuit

- $\quad$ Driver circuit

- Inverter circuit

\subsection{Power Supply Circuit}

A power supply is a device that supplies electric power to an electrical load. In various parts of the circuit, $5 \mathrm{~V}$ and $12 \mathrm{~V}$ constant value supplies are required.

\subsection{Voltage regulator:}

The voltage source in a circuit may have fluctuations and would not give the fixed voltage output. The voltage regulator IC maintains the output voltage at a constant value.

\subsection{Control circuit:}

SPWM technique is used to generate gate pulses, this is achieved by comparison of a triangle wave with a reference signal, here a sine wave.

\subsubsection{Triangular wave generator:}

In case of SPWM technique, the gate pulses for MOSFET is produced by comparing high frequency triangular carrier wave and the sinusoidal reference wave. Here, we are using op-amp LM318 in order to generate the triangular wave.The triangular wave generation circuit using LM318 can be obtained by the combination of a Schmitt trigger and an Integrator.

\subsubsection{Comparator:}

A comparator is a device that compares two voltages or currents and outputs a digital signal indicating which is larger. It has two analog input terminals, $\mathrm{V}+$ and $\mathrm{V}$-. It also has one binary digital output Vo. When the reference wave is greater than the carrier wave the pulse is generated.

\subsection{Isolation Circuit}

Isolation circuit is used between the control circuit and power circuit as devices in control circuit are not capable of handling voltages greater than $24 \mathrm{~V}$, whereas the power circuit operates at a voltage of around $110 \mathrm{~V}$ or more. For this purpose an optocoupler is used.

An opto-isolator, also called an optocoupler, photocoupler, or optical isolator, is a component that transfers electrical signals between two isolated circuits by using commercially available opto-isolators withstand input-to-output voltages up to $10 \mathrm{KV}$ and voltage

An opto-isolator contains a source of light, almost always a near infrared light-emitting diode (LED), that converts electrical input signal into light, a closed optical channel, and a photo sensor, which detects incoming light and either generates electric energy directly, or modulates electric current flowing from an external power supply. Opto-isolator can transfer the light signal not transfer the electrical signal. The sensor can be a photo resistor, a photodiode, a phototransistor.

\subsection{Driver Circuit}

A gate driver is a power amplifier that accepts a low-power input from a controller IC and produces a high-current drive input for the gate of a high-power transistor such as an IGBT or power MOSFET.

The switching signal for a transistor is usually generated by a logic circuit or a microcontroller, which provides an output signal that typically is limited to a few mill amperes of current. Consequently, a transistor which is directly driven by such a signal would switch very slowly, with correspondingly high power loss. During switching, the gate capacitor of the transistor may draw current so quickly that it causes a current overdraw in the logic circuit or microcontroller, causing overheating which leads to permanent damage or even complete destruction of the chip. To prevent this from happening, a gate driver is provided between the microcontroller output signal and the power transistor.

\subsection{Inverter Circuit}

A power inverter, or inverter, is an electronic device or circuitry that changes direct current (DC) to alternating current (AC). A typical power inverter device or circuit will require a relatively stable DC power source capable of supplying enough current for the intended overall power handling of the inverter. 
Three-phase inverters are used for variable-frequency drive applications and for high power applications. A basic three-phase inverter consists of three single-phase inverter switches each connected to one of the three load terminals. For the most basic control scheme, the operation of the three switches is coordinated so that one switch operates at each 60 degree point of the fundamental output waveform. When carrier-based PWM techniques are applied to six-step waveforms, the basic overall shape, or envelope, of the waveform is retained so that the 3 rd harmonic and its multiples are cancelled.

\subsection{Hardware setup}

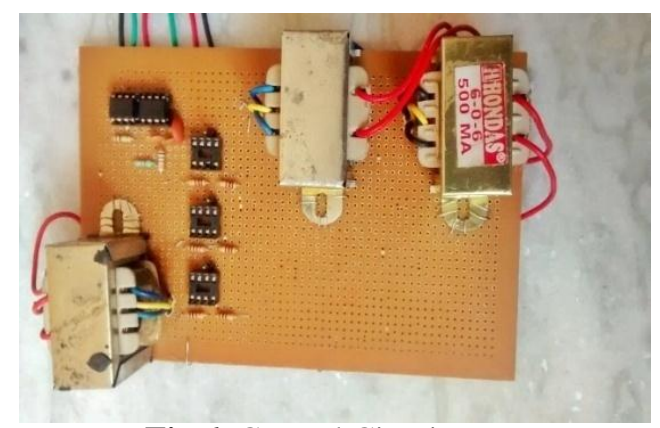

Fig 6. Control Circuit.

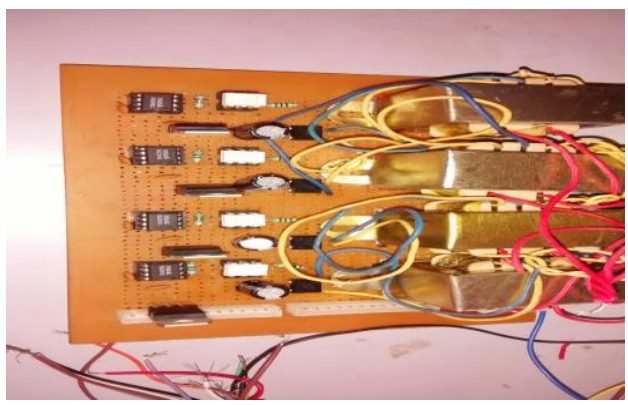

Fig8. Isolation and Driver Circuit.

\subsection{Hardware output}

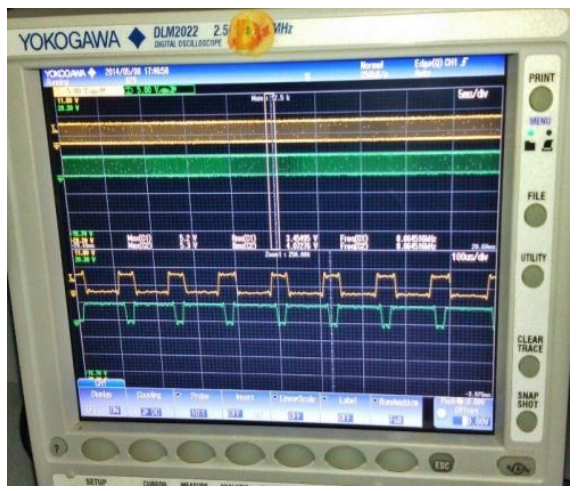

Fig9. Output Gate pulses.

\subsection{Hardware Inverter output}

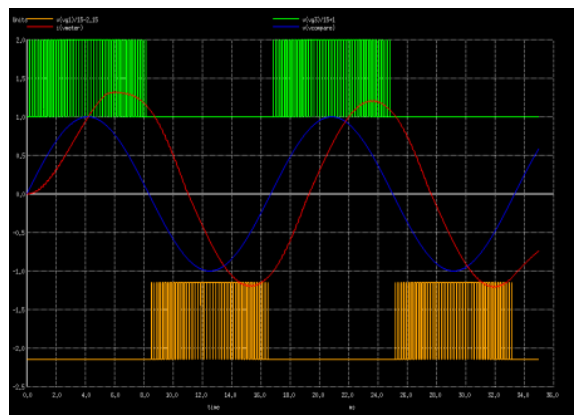

Fig10. Inverter Output. 


\section{Conclusion and Future work}

As seen from the above discussion SPWM is superior as compared to pulse width modulation in many aspects like:

1) The Modulation Index is higher for SPWM as compared to PWM

2) The output voltage is about $15 \%$ more in case of SPWM as compared to PWM.

3) The current and torque harmonics produced are much less in case of PWM.

Hence we see that there is a certain trade off that exists while using SPWM for inverters for Adjustable speed Drive Operations. Due to this we have to choose carefully as to which of the two techniques to use weighing the pros and cons of each method.

\section{References}

[1]. Prachi S. Dharmadhikari and Gaurav N. Goyal "Analysis \& Hardware Implementation of Three-Phase Voltage Source Inverter".

[2]. Nazmul Islam Raju, Md. Shahinur Islam, Tausif Ali,Syed Ashraful Karim "Study of SPWM Technique \& Simulation of Designed Analog Circuit (Op-Amp) Controlled Three Phase PWM Inverter with Harmonic Reduction".

[3]. Zhang Housheng, Zhao Yanlei and Li Haidong "Design of Three-phase Intermediate Frequency Aviation Power Based on Single Chip Microcomputer",

[4]. Lin Chengwu,Liu Yan and Sun Bingbin "Study On The Technology Of Inverter Based On DSP And SPWM".

[5]. Yaow-Ming Chen, Member, IEEE, Chih-Hung Hsieh, and Yuan-Ming Cheng

[6]. "Modified SPWM Control Schemes for Three-phase Inverters".

[7]. Mahesh A. Patel, Ankit Patel, Dhaval R.Vyas and Ketul Patel "Use of PWM Techniques for power Quality" International Journal of Recent Trends in Engineering, Vol. 1, No. 4, May 2009" 